\title{
HUman MicroNucleus Project: International Database Comparison for Results With the Cytokinesis-Block Micronucleus Assay in Human Lymphocytes: I. Effect of Laboratory Protocol, Scoring Criteria, and Host Factors on the Frequency of Micronuclei
}

\author{
Stefano Bonassi, ${ }^{1 *}$ Michael Fenech, ${ }^{2}$ Cecilia Lando, ${ }^{1}$ Yi-ping Lin, ${ }^{3}$ \\ Marcello Ceppi, ${ }^{1}$ Wushou Peter Chang, ${ }^{3}$ Nina Holland, ${ }^{4}$ \\ Micheline Kirsch-Volders, ${ }^{5}$ Errol Zeiger, ${ }^{6}$ Sadayuki Ban, ${ }^{7}$ Roberto Barale, ${ }^{8}$ \\ Maria Paola Bigatti, ${ }^{9}$ Claudia Bolognesi, ${ }^{10}$ Cao Jia, ${ }^{11}$ Marina Di Giorgio, ${ }^{12}$ \\ Lynnette R. Ferguson, ${ }^{13}$ Aleksandra Fucic, ${ }^{14}$ Omar Garcia Lima, ${ }^{15}$ \\ Patrizia Hrelia, ${ }^{16}$ Ayyathan P. Krishnaja, ${ }^{17}$ Tung-Kwang Lee, ${ }^{18}$ \\ Lucia Migliore, ${ }^{8}$ Ludmilla Mikhalevich, ${ }^{19}$ Ekaterina Mirkova, ${ }^{20}$ \\ Pasquale Mosesso, ${ }^{21}$ Wolfgang-Ulrich Müller, ${ }^{22}$ Youichi Odagiri, ${ }^{23}$ \\ Maria Rosaria Scarfi, ${ }^{24}$ Elena Szabova, ${ }^{25}$ Irena Vorobtsova, ${ }^{26}$ Anne Vral, ${ }^{27}$ \\ and Andrea Zijno ${ }^{28}$ \\ 'Department of Environmental Epidemiology, Istituto Nazionale per la Ricerca sul \\ Cancro, Genoa, Italy \\ ${ }^{2}$ Commonwealth Scientific and Industrial Research Organization (CSIRO) Health \\ Sciences and Nutrition, Adelaide, Australia \\ ${ }^{3}$ Institute of Environmental Health Sciences, National Yang Ming University Medical \\ School, Taipei, Taiwan, Republic of China \\ ${ }^{4}$ School of Public Health, University of California, Berkeley, California \\ ${ }^{5}$ Laboratory for Cell Genetics, Vrije Universiteit Brussels, Brussels, Belgium \\ ${ }^{6}$ Environmental Toxicology Program, National Institute of Environmental Health \\ Sciences, Research Triangle Park, North Carolina \\ ${ }^{7}$ Department of Radiobiology, Radiation Effects Research Foundation, Hiroshima, Japan \\ ${ }^{8}$ Dipartimento di Scienze dell'Uomo e dell'Ambiente, University of Pisa, Pisa, Italy \\ ${ }^{9}$ Dipartimento di Biologia Animale, University of Turin, Turin, Italy \\ ${ }^{10}$ Toxicological Evaluation Unit, Istituto Nazionale per la Ricerca sul \\ Cancro, Genoa, Italy \\ ${ }^{1}$ Molecular Toxicology Laboratory, Third Military Medical University, Chong Qing, China \\ ${ }^{12}$ Laboratorio Dosimetria Biologica, Autoridad Regulatoria Nuclear, Buenos Aires, Argentina \\ ${ }^{13}$ Auckland Cancer Society Research Centre, Auckland, New Zealand \\ ${ }^{14}$ Institute for Medical Research end Occupational Health, Zagreb, Croatia \\ ${ }^{15}$ Centro de Proteccion e Higiene de las Radiaciones, Havana, Cuba \\ ${ }^{16}$ Department of Pharmacology, University of Bologna, Bologna, Italy \\ ${ }^{17}$ Cell Biology Division, Bhabha Atomic Research Center, Mumbai, India \\ ${ }^{18}$ Department of Radiation Oncology, Leo Jenkins Cancer Center, East Carolina \\ University School of Medicine, Greenville, North Carolina \\ ${ }^{19}$ Institute of Genetics and Cytology, Belarus Academy of Sciences, Minsk, Belarus \\ ${ }^{20}$ Nacional Center of Hygiene, Medical Ecology and Nutrition, Sofia, Bulgaria \\ ${ }^{21}$ Dipartimento di Agrobiologia e Agrochimica, Università della Tuscia, Viterbo, Italy \\ ${ }^{22}$ Institute für Medizinische Strahlenbiologie, Universitatsklinikum Essen, Essen, Germany \\ ${ }^{23}$ Division of Human and Health Sciences, Yamanashi Prefectural College of \\ Nursing, Kofu, Japan \\ ${ }^{24}$ Consiglio Nazionale delle Ricerche (CNR)-Istituto Di Ricerca Per \\ I'Elettromagnetismo ei Componendi Elettronici (IRECE), Naples, Italy \\ ${ }^{25}$ Institute of Preventive and Clinical Medicine, Bratislava, Slovak Republic \\ ${ }^{26}$ Central Research Institute of Roentgenology and Radiology, St. Petersburg, Russia \\ ${ }^{27}$ Department of Anatomy, Embryology, and Histology, University Gent, Ghent, Belgium \\ ${ }^{28}$ Laboratory of Comparative Toxicology and Ecotoxicology, Istituto Superiore di \\ Sanità, Rome, Italy
}

